\title{
Philosophische Ethik: Fahne im Wind oder Fels in der Brandung?**
}

\author{
Der Präsident der Schweizer Nationalen Ethikkommission im Bereich der Human- \\ medizin und Lehrstuhlinhaber für Philosophie der Universität Tübingen unterschei- \\ det zwei Teile der Ethik: die Fundamentalethik, der es um den Begriff der Moral und \\ letzte Prinzipien moralischen Handelns geht, und die angewandte Ethik, die sich mit \\ moralischen Fragen einzelner Lebensbereiche, etwa der Medizinischen Ethik, befasst. \\ Für beide gilt, dass sie prinzipienstark sein sollten.
}

Otfried Höffe

\section{Moralische Grundsätze sind grösstenteils interkulturell und interepochal gültig}

\footnotetext{
* Der Text basiert auf einem Referat, das der Autor im März dieses Jahres im Rahmen der 6. Trendtage Gesundheit Luzern gehalten hat.
}

Korrespondenz: Prof. Dr. phil. Dres. h.c. Otfried Höffe Philosophisches Seminar Universität Tübingen Bursagasse 1 D-72070 Tübingen uni-tuebingen.de
Ethikkommissionen sollen aktuelle Probleme der Gesellschaft nach ethischen Gesichtspunkten beurteilen. Dabei droht die Gefahr, dass die Vertreter der (philosophischen) Ethik sich dem Zeitgeist anpassen und wie eine Fahne im Wind sich wechselnden Interessen und Moden unterwerfen. Tatsächlich darf man von ihnen erwarten, dass sie mittels ethischer Grundsätze couragiert genug sind, wo nötig, wie ein Fels in der Brandung, dem Zeitgeist Widerstand zu leisten. Daher Menschen lehren, sein Leben, von Schwierigkeiten unbeirrt, an unerschütterlichen Grundsätzen auszurichten? Die Antwort erfolgt in fünf Schritten. Zu Anfang überlege ich, was eine philosophische Ethik, danach, was eine ethische Innovation ist. Im dritten Schritt skizziere ich für Moral und Ethik so gut wie unwandelbare Grundsätze, im vierten, wie es sich bei einer Ethikberatung von der Art der Ethikkommissionen verhält. Und zum Schluss ziehe ich eine fraglos vorläufige Bilanz.

\section{Was ist Philosophische Ethik?}

Andere kommen gern rasch zur Sache; ein Philosoph fragt zunächst, was die Sache denn ist: Eine Ethik hat letztlich die Aufgabe, sowohl vorgegebene als auch alternative Verhaltensweisen auf ihre Berechtigung zu beurteilen. Die Grundsätze der berechtigten Verhaltensweisen fasst sie unter dem Ausdruck «kritische Moral» oder kürzer «Moral» zusammen. Des näheren besteht die Ethik aus zwei Teilen. Der erste Teil, ein Grundlagendiskurs, klärt den Begriff der Moral und begründet jene wahrhaft letzten Prinzipien moralischen
Handelns, - man kann sie Prinzipien zweiter Stufe nennen -, die noch grundlegender als die der Medizinethik vertrauten vier oder fünf Prinzipien (erster Stufe) sind: die Fürsorge (vgl. «salus alegroti»), das Nicht-Schaden («nil nocere»), die aufgeklärte Zustimmung (Autonomie) und in sozialer Perspektive die Gerechtigkeit, erweitert um Solidarität. Zum Grundlagendiskurs, der Fundamentalethik, gehört die Suche nach einem allgemeinen Massstab für die Moral, die Suche nach einem «Moralometer», das jedoch strukturell komplizierter als ein Thermometer ausfällt. Der zweite Teil, die sogenannte angewandte Ethik, befasst sich mit moralischen Fragen ausgewählter Lebensbereiche. Auch dann, beispielsweise als Medizinische Ethik, interessiert sich die Philosophie nicht für konkrete Einschätzungen. Schon gar nicht agiert sie wie Betty Bossi: Für moralisches Handeln stellt sie keine Rezepte bereit.

\section{Ethische Innovationen?}

Die so skizzierte Ethik versperrt sich nicht gegen Neuerungen, zumal dann nicht, wenn sie der entscheidenden Bezugsgrösse, dem Menschen, zugute kommen. Im Gegenteil verdienen Neuerungen, die dem Menschen dienen, den Ehrentitel der Innovation und sind der Ethik hochwillkommen. Innerhalb der Philosophie gelten sogar ausschliesslich jene Autoren als wirklich bedeutsam, denen tatsächlich eine ethische Innovation gelingt, also eine Innovation in der Theorie der Moral. Denn für Innovationen der Moral selbst ist die Ethik nur mitzuständig, nicht alleinzuständig.

Nun lehrt die Erfahrung, dass moraltheoretische Innovationen nicht im Tempo wissenschaftlicher oder technischer Innovationen erfolgen. Nicht in der Geschwindigkeit weniger Jahrzehnte oder sogar einzelner Jahre findet sie statt. Für wahrhafte Innovationen braucht es, was unsere hektische Zeit verlernt hat; es bedarf eines Atems von Jahrhunderten. Und blickt man auf den Gegenstand der Ethik, die moralischen Grundsätze, so ist ein erheblicher Teil davon der 
Menschheit seit Jahrtausenden bekannt: Moralische Grundsätze sind grösstenteils interkulturell und interepochal gültig.

\section{Anerkannte Grundsätze}

Manche Philosophen vertreten allerdings eine Gegenposition, den Relativismus. Ihm zufolge herrschen in den verschiedenen Kulturen und Epochen so unterschiedliche Vorstellungen vom Guten und Gerechten, dass die These der interkulturellen Übereinstimmung falsch sein muss.

Diese Gegenposition ist seit langem bekannt und hat sich trotzdem nicht durchgesetzt. Schon antike Denker kennen den Grundsatz: andere Länder, andere Sitten. Auf die Frage, wie man mit den Verstorbenen angemessen umgehe - berichtet der griechische Geschichtsschreiber Herodot - sagen die Inder: wir verbrennen sie, die Perser: wir essen sie, und die Griechen: wir bestatten sie (Historien 3, 38.2 - 4).

Diese Unterscheidungen sind eklatant, gewiss. Lässt man sich vom ersten Eindruck nicht verblüffen, so sieht man jedoch, dass sie relativ konkrete Verbindlichkeiten betreffen. Auf der moralisch entscheidenden Ebene, der Fundamentalebene, findet sich dagegen ein erstaunliches Mass an Gemeinsamkeiten.

Bei der Behandlung der Toten zeigen sie sich in der Hochachtung vor ihnen. Die Gemeinsamkeiten reichen aber noch weiter: So gut wie alle Kulturen anerkennen ein Lügeverbot und den Schutz des Lebens. Eine hohe Wertschätzung finden auch moralische Grundhaltungen wie Besonnenheit, Gerechtigkeit und Fairness, wie Aufrichtigkeit, Hilfsbereitschaft und Courage. Diese Gemeinsamkeiten entdecken wir schon bei so weit zurückliegenden Kulturen wie AltÄgypten, Alt-Indien und Alt-China. Weiterhin schätzt man allerorten einen moralischen Grundsatz, den der Wechselseitigkeit, die Goldene Regel, etwa ihre negative Fassung: «Was Du nicht willst, dass man Dir tu, das füg' auch keinem anderen zu.»

Dieses hohe Mass an interkultureller Gemeinsamkeit erlaubt es, von einem Weltmoralerbe zu sprechen: Über allen Unterschieden, die die Menschen trennen, über der Verschiedenheit der Sprachen, Sitten und Gebräuche sowie der Religionen und Konfessionen, dürfen wir jedenfalls nicht übersehen, dass sich die Menschen in der für sie wichtigen Angelegenheit weithin einig sind: in den als moralisch eingeschätzten Grundhaltungen.

Zur ernsthaften Philosophie gehört allerdings die Auseinandersetzung mit der Skepsis. Die hier entscheidende Skepsis räumt die genannten Gemeinsamkeiten ein, erklärt aber, die Prinzipien zweiter Stufe seien doch umstritten. In der Tat setzen einige Moralphilosophen, die sogenannten Utilitaristen, auf das Prinzip des grössten Glücks der grössten Zahl. Dagegen richtet Friedrich Nietzsche einen seiner «Sprüche und Pfeile» aus der Götzen-Dämmerung (Nr. 12): «Der Mensch strebt nicht nach Glück, nur der Engländer tut das.»
Heute nimmt selbst der englische Sprachraum von dem lange vorherrschenden Utilitarismus Abstand. Seit John Rawls, also mittlerweile seit fast vier Jahrzehnten, beruft er sich lieber auf den bedeutendsten Moralphilosophen der Neuzeit, auf Immanuel Kant. Die Gründe, die gegen den Utilitarismus und für Kant sprechen, liegen zum Teil schon im Begriff der Moral. Dessen Bestimmung findet aber in einem philosophischen Binnendiskurs statt, deren Grundzüge eine angewandte Ethik zur Kenntnis nimmt, ohne sich selbst auf den Binnendiskurs einzulassen. Stattdessen knüpft sie, zum Beispiel als Medizinische Ethik, an allgemein anerkannte Prinzipien an. Dazu gehören die oben genannten vier oder fünf Prinzipien (erster Stufe) und, ihnen in systematischer Hinsicht noch vorgelagert, der Gedanke der unantastbaren Menschenwürde und die Menschenrechte.

Eine kluge angewandte Ethik folgt also dem Vorbild guter Richter und guter Gesetzgeber. Sie orientiert sich nicht an privaten Grundsätzen. Gemäss einer Methode, die auf einen der grössten Moralphilosophen, auf Aristoteles, zurückgeht, beruft sie sich so weit wie möglich auf unkontroverse Grundsätze. Sie argumentiert, sagt man, topisch.

Diese Methode ist nicht etwa an Aristoteles' Zeiten gebunden. Im Gegenteil passt sie sehr gut für unsere Epoche. Sie begründet nämlich eine Ethik für freie Bürger, des näheren für freie Bürger einer säkularen und pluralistischen, spätestens im globalen Massstab auch multikulturellen Welt. Einzelne Gruppen dürfen sich nämlich auf ihre religiösen Überzeugungen berufen. Die moderne Gesellschaft als ganze zeichnet sich aber durch eine Vielfalt von Bekenntnissen und Religionen, zudem von Weltanschauungen und Wertorientierungen aus, welche die Indifferenz gegen alle Weltanschauungen und Religionen, sogar deren ausdrückliche Ablehnung einschliessen. Zu Recht erwarten daher freie Bürger von ihrem Gemeinwesen, dass dessen Rechtsordnung sich auf eine allgemeine Menschenvernunft beruft. Freie Bürger billigen diese allerdings nicht nur sich selbst, sondern allen Menschen zu. Sie rechnen deshalb mit allgemein anerkannten Grundsätzen.

Für deren Begründung ist vor allem der Weltbürger aus Königsberg, Immanuel Kant, wichtig. Seine Begründungsstrategie kann man in sechs Sätzen zusammenfassen. Erster Satz: Moralische Verbindlichkeiten gelten in dem Sinn uneingeschränkt - Philosophen sagen: kategorisch -, dass sie sich gegen vor- und aussermoralische Verbindlichkeiten nicht verrechnen lassen. Im Namen wirtschaftlicher Vorteile beispielsweise darf man keinen Unschuldigen bestrafen. Zweiter Satz: Die wahrhaft moralischen Verbindlichkeiten gelten für alle Menschen jedweder Kultur; die Moral ist in ihrem Kern universalistisch. Dritter Satz: Zur universalistischen Moral gehört aufseiten der Richtigkeit («objektive Seite») die strenge Verallgemeinerbarkeit von Handlungsgrundsätzen, Maximen genannt. Vier- 
ter Satz: Als Prinzip des moralischen Subjekts («subjektive Seite») gilt die Selbstgesetzlichkeit (Autonomie) des Willens. Fünfter Satz: Im Gegensatz zum Utilitarismus sind Folgenüberlegungen nur handlungsintern, nicht handlungsextern zulässig. Bei der Hilfspflicht beispielsweise muss man überlegen, welches Tun oder Lassen die einschlägige Hilfe verspricht. Man darf jedoch nicht fragen, welche positiven Folgen die Hilfsbereitschaft für mein Wohlergehen hat, beispielsweise für meine Geschäftsinteressen, für die Erwartung einer finanziellen oder sozialen Belohnung (Ansehen). Sechster und letzter Satz: Die unantastbare Menschenwürde stellt samt den daraus fliessenden Menschenrechten das inhaltliche Leitprinzip jedes moralischen Lebens und Zusammenlebens dar. niemand hält es für moralisch zulässig, im Namen eines noch so grossen Kollektivwohls einen Unschuldigen zu bestrafen oder eine missliebige Person zu erschlagen.

Der Utilitarist Peter Singer dagegen kennt kein absolutes Tötungsverbot. Stattdessen setzt er sich grosszügig für eine Rechtfertigung von Abtreibung, Kindstötung und nichtfreiwilliger Euthanasie ein. Beispielsweise sei sehr oft die Tötung eines Kindes «kein Unrecht». Selbst im Utilitarismus werden aber weder die Moral noch die Ethik zur Fahne im Wind. Das Prinzip (zweiter Stufe), das maximale Kollektivwohl, bleibt nämlich unangefochten. Bei einer Form, dem Regelutilitarismus, sind auch die moralischen Regeln so gut wie unerschütterlich. Und beim Handlungsutilitaris-

\section{Der Utilitarismus erlaubt soziale und rechtliche Diskriminierungen, etwa die Bestrafung eines Unschuldigen, vorausgesetzt, die kollektive Glücksbilanz wird maximiert}

Offensichtlich sind nach all diesen Sätzen weder die Moral noch deren Theorie, die Ethik, Fahnen im Wind. Viel eher sind sie ein Fels, der auch starker Brandung standhält.

Merkwürdigerweise überlebt der Utilitarismus, im wesentlichen aber nur in der angewandten Ethik. Ein Peter Singer vertritt ihn, freilich ohne sich mit Rawls' Kritik angemessen auseinanderzusetzen. Der Utilitarismus erscheint deshalb vielen als attraktiv, weil er zwei starke und je für sich plausible Antriebskräfte zu einer Einheit zu bringen verspricht: das Interesse am eigenen Wohl und die moralische Forderung, das Wohl der anderen mitzuberücksichtigen. Eine erste Schwierigkeit des Utilitarismus liegt in der Verschiedenartigkeit dieser zwei Bestandteile. Wer dem fremdem Wohl dienen will, muss bisweilen sein eigenes Wohl einschränken. Da nun im Konfliktfall stets das Wohl aller Betroffenen vorrangig sein soll, verlangt der Utilitarismus ein unrealistisch hohes Mass an Altruismus. Weiterhin lässt er die Frage offen, wie beim Wohl aller die einzelnen Betroffenen zu berücksichtigen sind. Da das Gesamtwohl zu maximieren ist, darf der Nachteil der einen gegen den Vorteil der anderen verrechnet werden. Infolgedessen erlaubt der Utilitarismus soziale und rechtliche Diskriminierungen, beispielsweise die Bestrafung eines Unschuldigen. Vorausgesetzt, dass die kollektive Glücksbilanz maximiert wird, reflektiert er sogar eine so verwerfliche Institution wie die Sklaverei.

Weiterhin erklärt der Gründungsvater, Jeremy Bentham, die rechtsmoralische Errungenschaft der Neuzeit, die Menschenrechte, zum «gestelzten Unsinn». Dem widerspricht die neuzeitliche Moral vehement. Ihre Rechtsmoral hält es für selbstverständlich, dass der Mensch, bloss weil er Mensch ist, angeborene und unveräusserliche Rechte besitzt. So gut wie mus passt man sich nicht etwa den jeweiligen Situationen an. Wohl aber ist die Situation im Lichte des Kollektivwohls zu bewältigen.

Im übrigen folgen die genannten Moralvorstellungen nicht aus Singers Utilitarismus, sondern aus seinem Vernunftbegriff. Als Person gilt bei ihm jedes seiner selbst bewusste bzw. rationale Wesen, für Singer einschliesslich der Schimpansen, Gorillas, Wale und Delphine. Dagegen zählen weder Embryonen und Föten noch Neugeborene und Kleinkinder, es sei denn die Eltern erheben Einspruch. Auch Erwachsene, die durch Unfall, Krankheit oder hohes Alter ihren Status als selbstbewusstes oder rationales Wesen verlieren, werden nicht mehr als Personen anerkannt. Humanerweise fiel Singer aber in einen pragmatischen Widerspruch. Er sah nämlich in seiner an Alzheimer erkrankten Mutter eine Person und liess ihr eine Rund-umdie-Uhr-Pflege zukommen. Erwarten sollte man daher, dass er diesen pragmatischen Widerspruch überwindet, indem er seine moralphilosophische Position grundlegend ändert.

\section{Ethikkommissionen}

Auf allen drei Ebenen: bei der Moral, beim philosophischen Grundlagendiskurs und bei der darauf aufbauenden angewandten Ethik, gibt es so gut wie unwandelbare Grundsätze. Wie verhält es sich bei den Ethikkommissionen? Ihre Aufgabe besteht darin, drei grundverschiedene Elemente aufeinander zu beziehen und miteinander zu vermitteln.

Das erste Element, die moralischen Grundsätze, sind wie gesagt seit langem anerkannt. Das zweite Element besteht in Sachgesetzlichkeiten, etwa in Grundkenntnissen von Medizin, ihrer Forschung und der Organisation eines Krankenhauses. Und als drittes Ele- 
ment braucht es Einsichten in die aktuelle Lage, insbesondere in deren Schwierigkeiten.

Diese drei Elemente sind nicht etwa schlicht zu addieren. Für die Ethikberatung genügen daher nicht Kenntnisse. Es braucht zusätzlich eine kreative Urteilsfähigkeit. Man hat nämlich die anfallenden Probleme wahrzunehmen und sie im Licht der moralischen Grundsätze aufzuarbeiten.

Bei dieser Arbeit braucht sich die Ethik zwar nicht zu einer Windfahnen-Ethik verführen und entwürdigen lassen, ihre Prinzipienfestigkeit ist aber gefährdet. Die Gefährdung resultiert beispielsweise aus einem moralischen Mantel, den man sich gern umhängt, der «humanitären Ethik» des Heilens und Helfens. Unter diesem Deckmantel berufen sich manche Ethikvertreter auf eine «schuldhafte Verstrickung». Sie behaupten, man befinde sich in dem «tragischen Dilemma», entweder an A, beispielsweise an heutigen menschlichen Embryonen, oder aber an B, an zukünftigen Patienten, schuldig zu werden. Denn an Embryonen würde zwar eine Forschung vorgenommen, die für sich betrachtet moralisch nicht unbedenklich sei. Diese Forschung eröffne aber Diagnose- und Therapiemöglichkeiten für künftige Patienten, die man ihnen beim Verbot verbrauchender Embryonenforschung verweigere.

\section{Den humanitaristischen Fehlschluss begeht, wer den grundsätzlichen Vorrang verkennt, den der Lebensschutz vor dem Hilfsgebot geniesst}

Diesem Rechtfertigungsversuch liegt jedoch ein Fehlschluss zugrunde. Wegen der Berufung auf die «humanitäre» Ethik des Heilens und Helfens nenne ich ihn den «humanitaristischen Fehlschluss». In ihrer moralisch gravierendsten Gestalt verkennt sie das unterschiedliche Gewicht der moralischen Verpflichtungen. Schon die Alltagsmoral unterscheidet eine unverzichtbare Elementarmoral von der moralischen Mehrleistung. Die philosophische Ethik bringt diese Unterscheidung auf den Begriff: Während die Anerkennung der Elementarmoral, der sogenannten Rechtspflichten, den Mitmenschen geschuldet ist, sie deshalb notfalls mit Zwang durchgesetzt werden darf, reichen die Mehrleistungen der sogenannten Tugendpflichten über das Geschuldete hinaus in den Bereich des Freiwilligen.

Zur zwangsbefugten Elementarmoral gehören nun die strengen Verbote zu stehlen, zu betrügen und zu töten, zur freiwilligen Mehrleistung dagegen das Gebot der Wohltätigkeit. Dabei geniesst die Rechtsmoral, da ihre Anerkennung geschuldet ist, den kompromisslosen Vorrang. In Übereinstimmung mit unseren moralischen Grundintuitionen darf man im Namen der Wohltätigkeit andere Menschen weder bestehlen noch betrügen oder gar töten. Insbesondere freie Bürger lassen sich weder in ihren Rechten beschneiden, noch greifen sie in die entsprechenden Rechte ihrer Mitbürger ein. Sie pochen vielmehr wechselseitig auf ihre Rechte und behalten sich die Freiheit, aber nicht den Zwang zur Wohltätigkeit vor.

Den humanitaristischen Fehlschluss begeht nun, wer den grundsätzlichen Vorrang verkennt, den der Lebensschutz vor dem Hilfsgebot geniesst. Das ärztliche Ethos spielt auf diesem Vorrang mit dem zweiten hippokratischen Grundsatz an: "primum nil nocere»: Als erstes darf man nicht schädigen. Ein Patient könnte so töricht sein, unter Berufung auf seine Selbstbestimmung eine medizinisch nicht gebotene Amputation zu erbitten. Wer dagegen durch die Spende einer Niere jemandem das Leben rettet, ohne sein eigenes zu gefährden, handelt nicht töricht, sondern hilfsbereit; er ist wohltätig. Während der Arzt dem Hilfsbereiten zu Hilfe kommen sollte, darf er auf keinen Fall einem Dritten Schaden zufügen. Gemäss einem weiteren Grundsatz medizinischer Ethik, der Selbstbestimmung (Autonomie), darf er niemandem ohne Zustimmung eine Niere herausoperieren.

\section{Bilanz}

An dieser Stelle breche ich meine Überlegungen ab und ziehe Bilanz: Philosophen, die ihre Fahne nach dem Winde drehen, mag es geben. Bei einigen kann es aus dem gewiss unehrenhaften Grund geschehen, Beifall vom Zeitgeist zu erhalten, also aus einem Zeitgeistopportunismus. Anderen fehlt es an Courage, teils an intellektuellem, teils an charakterlichem Mut, sich dem Zeitgeist zu widersetzen. Wieder anderen mangelt es schlicht an moralischen und ethischen Grundsätzen. Nur sehr wenige Windfahnenphilosophen dagegen tun es aus moralphilosophischer Überzeugung, nämlich aus einem ethischen Relativismus. Gegen ihn, habe ich gezeigt, sprechen aber gute Argumente. Infolgedessen ist die philosophische Ethik gut beraten, prinzipienstark und prinzipientreu zu sein.

Auf der Ebene der Grundsätze hat die Ethik sogar ein noch grösseres Gewicht als der im Titel genannte Fels in der Brandung. Nach dem sprichwörtlichen Grundsatz «steter Tropfen höhlt den Stein» ist nämlich selbst dieses harte Material, Felsgestein, gegen Erosionen nicht gefeit. Wird der Tropfen zur Welle und die Welle zur aggressiven Brandung, so treten die Erosionen noch rascher und stärker auf.

Daher mein Schlusswort: Eine prinzipienstarke Ethik widersetzt sich nicht bloss der Windfahnenhaltung. Sie verhält sich sogar noch unerschütterlicher als ein Fels in der Brandung.

Zur selben Thematik folgt im September ein Beitrag von Johannes Fischer mit einer Replik von Otfried Höffe.

\section{Weitere Auseinandersetzungen mit der Literatur in:}

1 Höffe O. Medizin ohne Ethik? Frankfurt a.M.: Suhrkamp; 2003. Besonders Kap. 2.

2 Höffe O. Lebenskunst und Moral. Oder: Macht Tugend glücklich? München: Beck; 2007. Besonders Teil I. 